antigens is thought to have a central role in disease pathogenesis. Of particular interest is vimentin, a synovial antigen to which antibodies towards its citrullinated form are commonly found in both RA sera and synovial fluid. Anti-citrullinated vimentin antibodies are highly specific for RA patients and tightly associate with RA-predisposing genes, in particular with the HLA-DRB1*04 alleles, DRB1*0401 and DRB1*0404. Thus, citrullinated (potentially arthritogenic) peptides from vimentin may be presented by $\mathrm{MHC}$ class II to autoreactive $\mathrm{T}$ cells and evoke anti-citrulline immune responses which could precipitate the development of RA.

Objective To identify peptides from citrullinated vimentin that specifically bind HLA-DRB1*0401 and to further study $T$ cell responses to citrullinated-vimentin in HLA-DRB1*0401 RA patients and healthy subjects.

Methods Using computer algorithms, the authors first identified a potential $T$ cell epitope from citrullinated vimentin followed by binding assays to empirically examine its affinity to HLA-DRB1*0401. The peptides were further validated in HLA-DRB1*0401 transgenic mice and citrullinated vimentinspecific $T$ cell responses were further monitored in humanised mice, RA patients and healthy controls using MHC class II tetramers and functional assays.

Results Only the citrullinated but not the native form of the vimentin peptide bound to HLA-DRB1*0401. Likewise, recall T cell responses were detected only in HLA-DRB1*0401 transgenic mice immunised with citrullinated vimentin in which specific CD4 T cells were also monitored using MHC class II tetramers. Furthermore, CD4 T cells from HLA-DR*0401 RA patients secreted tumour necrosis factor $\alpha$, interferon $\gamma$ and interleukin 17 upon stimulation with the citrullinated vimentin peptide.

Conclusions Based on computer analysis followed by mouse and human immunology, the authors describe a post-translational modification of a RA candidate autoantigen. The authors show DRB1*0401-restricted proinflammatory $T$ cell responses to a novel $T$ cell epitope on modified vimentin, a candidate autoantigen for $\mathrm{RA}$.

\title{
A185 IDENTIFICATION AND FUNCTION OF T CELLS REACTIVE WITH CITRULLINATED VIMENTIN IN HLA-DRB1*0401 HUMANISED MICE AND RHEUMATOID ARTHRITIS PATIENTS
}

O Snir, M Rieck, K Klich, L Klareskog, J H Buckner, V Malmström Department of Medicine, Rheumatology Unit, Karolinska University Hospital, Karolinska Institute, Solna, Stockholm, Sweden

10.1136/ard.2010.129668v

Background The pathological mechanisms leading to a lost of immunological tolerance and inciting rheumatoid arthritis (RA) are not fully understood. However, autoimmunity towards several post-translationally modified (citrullinated) synovial 